\title{
Fuentes de estrés percibidas y rendimiento académico de estudiantes de odontología chilenos
}

\author{
Clara Misrachi-Launert, Matías Ríos-Erazo, José M. Manríquez-Urbina, Cristián Burgos-Ibarra, \\ Daniela Ponce-Espinoza
}

Objetivo. Evaluar la influencia de distintas fuentes de estrés en el rendimiento académico de estudiantes de odontología de la Universidad de Chile.

Sujetos y métodos. Se utilizó el cuestionario de estrés en el ambiente dental (DESQ) modificado de 25 preguntas, que se aplicó al $60 \%$ de los estudiantes de cada año. Se compararon distintas fuentes de estrés percibidas entre los cursos. La prueba $r$ de Pearson se utilizó para determinar la influencia de las fuentes de estrés en el rendimiento académico.

Resultados. Una muestra de 302 estudiantes respondió el cuestionario. Entre los resultados se identificó que las principales fuentes de estrés en todos los cursos eran las calificaciones y los exámenes, el miedo a fallar en un curso o un año y la falta de tiempo para relajarse. Un factor de estrés importante para los cursos superiores fue la atmósfera negativa creada por los supervisores clínicos. La carga de trabajo presentó una correlación negativa con el rendimiento académico, mientras que la práctica preclínica y clínica mostraron una correlación positiva.

Conclusiones. El cuarto año parece ser el más estresante. El contacto temprano con los pacientes y una mejor planificación curricular y administración deben ponerse en práctica para evitar el aumento del estrés de la formación clínica.

Palabras clave. Estrés académico. Estudiantes de odontología. Rendimiento académico.

\section{Perceived sources of stress and academic performance of Chilean dental students}

Aim. To assess the influence of perceived sources of stress amongst University of Chile dental students on their academic performance.

Subjects and methods. In this research was used a modified Dental Environment Stress Questionnaire (DESQ) consisting of 25 questions was applied to $60 \%$ of students of each year. Tests were applied to compare perceived sources of stress between courses. The Pearson $r$ test was used to determine the influence of stress sources on academic performance.

Results. A sample of 302 students answered the questionnaire. In results, the main sources of stress in all courses were grades and examinations, fear of failing a course or a year and lack of time to relax. An important stressor for higher courses was the negative atmosphere created by clinical supervisors. Workload showed a negative correlation with academic performance, and preclinical and clinical training showed a positive correlation.

Conclusions. The fourth year seems being the most stressful. Earlier contact with patients and an improvement in curriculum planning and administration should be put in practice to avoid the increased stress of the clinical training.

Key words. Academic performance. Academic stress. Dental students.

\section{Introducción}

Numerosos estudios sobre las condiciones que afectan el bienestar mental de los profesionales muestran un alto grado de estrés entre los especialistas en odontología. Factores tales como la interacción diaria con los pacientes, colegas y personal auxiliar, combinado con problemas administrativos y la alta concentración requerida por las meticulosas tareas que deben realizarse en el reducido espacio bucal, dan lugar a estrés psicológico excesivo [1-5].
Además de la estresante vida profesional de los dentistas, los estudiantes de odontología deben soportar un ambiente de aprendizaje altamente exigente. Los planes de estudios de odontología actuales requieren la adquisición de conocimientos teóricos y competencias clínicas, incluyendo habilidades de comunicación [6]. El informe del comité para el cambio y la innovación de la Asociación Americana de Educación Dental [7] la describe como compleja, cara y profundamente insatisfactoria para el estudiante, caracterizada por programas de estudio
Departamento de Ciencias Básicas y Comunitarias. Facultad de Odontología. Universidad de Chile. Santiago, Chile

Correspondencia: Dr. José M. Manríquez Urbina. Departamento de Ciencias Básicas y Comunitarias. Facultad de Odontología. Universidad de Chile. Sergio Livingstone Pohlhammer, 943. Independencia, Santiago, Chile.

E-mail:

jmanriquezu@gmail.com

Conflicto de intereses: No declarado.

Competing interests: None declared.

(c) 2015 FEM 
Tabla I. Ítems del cuestionario de estrés dental en ambiente dental (DESQ), adaptado para la población de estudio.

1. Estrés debido a la cantidad de clases

2. Estrés debido a la dificultad de las clases

3. Estrés debido a las calificaciones y los exámenes

4. Estrés debido a la competencia con los compañeros

5. Estrés debido a la responsabilidad en el cuidado del paciente

6. Estrés debido a la dificultad de generar habilidades manuales de precisión en el trabajo preclínico y de laboratorio

7. Estrés debido a la dificultad en el aprendizaje de procedimientos y protocolos clínicos

8. Estrés debido a la falta de confianza en sí mismo

9. Estrés debido a la actitud del paciente hacia usted o hacia la odontología

10. Estrés debido a la atmósfera creada por los profesores clínicos

11. Estrés debido a la ausencia de docentes en preclínica o clínica

12. Estrés debido a la confianza en que los laboratorios entreguen los trabajos oportunamente

13. Estrés debido a la respuesta del personal administrativo y de apoyo:

secretarias (de departamentos y de estudios), asistentes en clínicas y de laboratorio central

14. Estrés debido a la relación entre compañeros

15. Estrés debido a reconsiderar la odontología como la elección apropiada de carrera

16. Estrés debido al miedo a suspender alguna asignatura

17. Estrés debido a las relaciones de pareja

18. Estrés debido a las responsabilidades financieras personales y universitarias

19. Estrés debido a que los pacientes no paguen sus tratamientos

20. Estrés debido a la falta de tiempo para relajarse

21. Estrés debido a la salud física personal y de otros miembros de la familia

22. Estrés debido a la relación con los padres y la familia

Instrucciones: el siguiente cuestionario busca identificar los estresores presentes entre los estudiantes de odontología y el nivel de estrés de cada uno de ellos. Se puntúan 1 (no existe un nivel de estrés), 2 (nivel bajo de estrés), 3 (nivel moderado de estrés), 4 (nivel alto de estrés) o 9 (no se aplica porque la fuente de estrés no se ajusta a su realidad).

inflexibles, sobrecargados y que promueven la memorización por encima del nivel de evidencia y el pensamiento crítico.

En un número importante de escuelas dentales en universidades europeas existe evidencia de que los estudiantes no disfrutan de sus experiencias de aprendizaje, por lo que la necesidad de cambio se ha convertido en el denominador común [8]. Un estudio realizados por David et al [9] concluye que el medio altamente estresante es lo que más contribuye a la insatisfacción de los estudiantes.

Es motivo de gran preocupación para los educadores que incluso en las primeras etapas de su proceso educativo los estudiantes de odontología muestran signos asociados con el síndrome de burnout, caracterizado por el agotamiento emocional, la despersonalización y sentimientos de escasos logros individuales [2-9]. Esta sintomatología, junto con el impacto del estrés en los procesos cognitivos, ha despertado el interés de realizar estudios sobre fuentes percibidas de estrés por los estudiantes de odontología y la influencia de éstas en el rendimiento académico [10].

Desde una aproximación interaccional, el término 'estrés' se define como la relación entre un individuo y el entorno, en el que algunas de sus características son percibidas como una amenaza para la salud física o mental [11]. Hay evidencia que documenta la etiología multifactorial del estrés en estudiantes de odontología, señalando como principales factores: los exámenes, el miedo al fracaso y los conflictos relacionados con las demandas de excesivo rendimiento hechas por la facultad [12-24].

Una de las consecuencias del estrés en los estudiantes es la disminución en el rendimiento académico [25-31]. En un estudio realizado con estudiantes de odontología de primer año, Westerman et al [6] comunicaron diferencias significativas en los niveles de ansiedad entre los estudiantes en el tercio superior de rendimiento académico y los del tercio más bajo, lo que sugiere una relación inversa entre ambas variables. Sanders y Lushington [10] estudiaron los efectos de la percepción de estrés en el desempeño académico de 202 estudiantes en una escuela australiana de odontología y demostraron que factores como los años de estudio del estudiante, la facultad y la administración pueden ser considerados predictores del rendimiento académico en estudiantes de pregrado de odontología.

En la Universidad de Chile, la duración del programa para cirujano dentista es de 12 semestres. Durante los primeros seis semestres, a los estudiantes se les enseña ciencia básica y odontología preclínica, mientras que entre el séptimo y décimo semestres se dedican al aprendizaje clínico. El undécimo semestre se dedica a un internado rotatorio realizado en la atención primaria de salud en centros rurales y urbanos y en hospitales pertenecientes al Ministerio de Salud Pública. En el último se- 
mestre, los estudiantes deben completar una investigación sobre distintos temas en los departamentos de la facultad; una vez aprobado, los estudiantes han de aprobar un examen de grado antes de recibir un título profesional. La cuota de pago mensual para el estudio de la odontología es una de las más altas de cualquier programa de pregrado, lo que lo hace aún más dificultoso; además, la mayoría de los estudiantes recién graduados se enfrentan a problemas financieros asociados con el coste de los programas de especialidad, que son extremadamente caros.

El objetivo de este estudio fue determinar las fuentes percibidas de estrés y su relación con el rendimiento académico de estudiantes de pregrado de odontología en la Universidad de Chile, la institución educativa más grande del país.

\section{Sujetos y métodos}

La población de estudio estuvo constituida por estudiantes de pregrado de primero a quinto año matriculados en la Facultad de Odontología de la Universidad de Chile. El estrés se midió en una muestra de 302 estudiantes $(61,4 \%$ del total) utilizando una forma modificada del cuestionario de estrés en ambiente dental (DESQ) [1] (Tabla I). El cuestionario consta de 25 preguntas aplicables a las diferentes situaciones que pueden ocasionar estrés en estudiantes en el marco de la educación dental en Chile. Las preguntas se agrupan en las siguientes seis categorías: carga de trabajo, formación preclínica y clínica, el tratamiento del paciente, de la facultad y de la administración, y relaciones interpersonales, entre otros. El cuestionario también incluye datos relativos a años de estudio y edad de los alumnos. Fue autoaplicado y se midió en una escala tipo Likert, con las siguientes opciones disponibles para la respuesta: 1 , no estresante; 2, poco estresante; 3 moderadamente estresante, y 4, muy estresante. Se obtuvo consentimiento escrito de los encuestados, al igual que la aprobación del comité de ética de la Facultad de Odontología.

Previamente a su aplicación, el DESQ fue validado y adaptado culturalmente para la población de estudio. En relación a la consistencia interna, el análisis estadístico mostró un $\alpha$ de Cronbach de 0,858 . Al final del proceso de validación se eliminaron tres ítems del cuestionario original, que quedó constituido finalmente por 22 ítems.

Las preguntas 6, 7, 9, 10,11, 12 y 13, relacionadas con el entrenamiento preclínico y clínico, se excluyeron del cuestionario aplicado a estudiantes de primer año. Asimismo, las preguntas 7, 8, 9 10, $11 \mathrm{y}$ 13 , relacionadas con la formación clínica, se exclu-
Tabla II. Distribución de la muestra por año de estudio y sexo.

\begin{tabular}{lccccc} 
& & \multicolumn{2}{c}{ Sexo } & & Media de \\
& Total & Hombres & Mujeres & $\begin{array}{c}\text { Tasa de } \\
\text { edad (años) }\end{array}$ & respuesta (\%) \\
\cline { 3 - 6 } Primer año & 81 & 37 & 44 & 18,6 & 82,60 \\
\hline Segundo año & 44 & 15 & 29 & 19,8 & 41,90 \\
\hline Tercer año & 59 & 17 & 42 & 21,3 & 61,45 \\
\hline Cuarto año & 66 & 28 & 38 & 23,6 & 64,07 \\
\hline Quinto año & 52 & 25 & 27 & 24,0 & 57,70 \\
\hline Total & 302 & 122 & 180 & 21,2 & 61,30 \\
\hline
\end{tabular}

yeron de los cuestionarios dados a los estudiantes de segundo año, ya que sólo se esperaba que fueran completadas por estudiantes con cursos preclínicos. El estudio se llevó a cabo a principios del segundo semestre del año académico 2009. Se pidió la participación a todos los estudiantes de la carrera, excepto los asistentes al programa de internado, que tiene lugar en el sexto año académico. Los cuestionarios se distribuyeron al comienzo de una de las clases en cada año de los estudios de pregrado. Se entregó una explicación del objetivo del estudio y las instrucciones para completar el cuestionario antes de iniciarlo. El tiempo máximo asignado para completar todos los ítems del cuestionario fue de 15 minutos. Todos los estudiantes participaron de manera voluntaria y no se intentó posteriormente incluir a aquellos que optaron por no participar.

La comparación entre el número previsto de horas semanales dedicadas a la práctica clínica y preclínica, como se indica en el programa oficial de capacitación, y el número real experimentado por los estudiantes se obtuvo a través de tres preguntas adicionales para ser respondidas por los cursos segundo a quinto. Estas preguntas investigaron acerca del número de horas dedicadas a las actividades preclínicas, incluidas las tareas de laboratorio dental: actividades unidas a la atención del paciente, tales como escribir historias clínicas, la preparación de los instrumentos para la esterilización y la realización de tareas de laboratorio dental.

El rendimiento académico se midió a través de la media de las calificaciones obtenidas por cada estudiante durante el semestre anterior. Los datos fueron adquiridos desde la oficina de pregrado. Tanto en la Universidad de Chile como en el resto del país, para la calificación de desempeño se usa una escala 
Tabla III. Fuentes de estrés percibidas durante la formación de pregrado en odontología.

\begin{tabular}{|c|c|c|c|c|c|c|c|}
\hline & Primer año & Segundo año & Tercer año & Cuarto año & Quinto año & Significación & Total \\
\hline \multicolumn{8}{|l|}{ Carga de trabajo } \\
\hline 2. Dificultades en entender las clases & $2,90 \pm 0,66$ & $2,48 \pm 0,76$ & $2,53 \pm 0,75$ & $2,18 \pm 0,88$ & $2,37 \pm 0,84$ & $1>2,4,5$ & $2,49 \pm 0,81$ \\
\hline 3. Exámenes y pruebas & $3,68 \pm 0,65$ & $3,57 \pm 0,62$ & $3,59 \pm 0,56$ & $3,38 \pm 0,72$ & $3,44 \pm 0,73$ & No significativo & $3,53 \pm 0,66$ \\
\hline 5. Pérdida de tiempo de descanso & $3,25 \pm 0,78$ & $3,00 \pm 1,06$ & $3,29 \pm 0,79$ & $3,15 \pm 0,92$ & $3,50 \pm 0,70$ & $5>2$ & $3,24 \pm 0,85$ \\
\hline Media & $3,19 \pm 0,47$ & $3,00 \pm 0,53$ & $3,15 \pm 0,53$ & $2,84 \pm 0,52$ & $3,05 \pm 0,63$ & $1,3>4$ & $3,04 \pm 0,55$ \\
\hline \multicolumn{8}{|l|}{ Práctica preclínica y clínica } \\
\hline $\begin{array}{l}\text { 6. Dificultades en la adquisición de } \\
\text { habilidades manuales de precisión }\end{array}$ & & $2,45 \pm 1,07$ & $3,32 \pm 0,88$ & $2,92 \pm 1,09$ & $2,77 \pm 1,11$ & $3>2,5$ & $2,87 \pm 1,04$ \\
\hline Media & & $2,45 \pm 1,07$ & $2,92 \pm 0,78$ & $2,86 \pm 0,83$ & $2,73 \pm 0,89$ & $3,4>2$ & $2,74 \pm 0,89$ \\
\hline \multicolumn{8}{|l|}{ Tratamiento de pacientes } \\
\hline 8. Responsabilidades en el cuidado del paciente & & & $1,69 \pm 1,15$ & $3,48 \pm 0,66$ & $3,37 \pm 0,79$ & $4,5>3$ & $2,85 \pm 0,87$ \\
\hline 9. Actitud del paciente hacia ti/dentista & & & $1,56 \pm 0,95$ & $2,59 \pm 0,91$ & $2,60 \pm 0,96$ & $4,5>3$ & $2,25 \pm 0,94$ \\
\hline $\begin{array}{l}\text { 10. Miedo de los pacientes a no } \\
\text { proporcionar su tratamiento }\end{array}$ & & & $1,24 \pm 0,73$ & $2,73 \pm 0,99$ & $2,88 \pm 0,78$ & $4,5>3$ & $2,28 \pm 0,83$ \\
\hline Media & & & $1,50 \pm 0,76$ & $2,93 \pm 0,64$ & $2,95 \pm 0,61$ & $4,5>3$ & $2,46 \pm 0,67$ \\
\hline \multicolumn{8}{|l|}{ Facultad y administración } \\
\hline Media & $1,67 \pm 0,94$ & $1,41 \pm 0,73$ & $2,61 \pm 0,75$ & $2,93 \pm 0,69$ & $2,97 \pm 0,64$ & $3,4,5>1,2$ & $2,32 \pm 0,75$ \\
\hline
\end{tabular}

numérica con un rango de 1,0-7,0. La calificación de aprobación mínima corresponde a 4,0. Las relaciones conceptuales generalmente utilizadas son las siguientes: 1 , muy malo; 2 , malo; 3 , menos de regular; 4 , normal; 5 , bueno; 6 , muy bueno, y 7 , excelente.

Para el perfil sociodemográfico, el nivel y fuentes de estrés, las horas dedicadas a las actividades del programa y el rendimiento académico se aplicaron estadísticas descriptivas. El comportamiento normal de las variables de estudio se confirmaron mediante la prueba de Kolmogorov-Smirnov.
El análisis de la varianza (ANOVA) y el test de Tukey post hoc se utilizaron para comparar las fuentes de estrés en los diferentes cursos. Una comparación de los niveles de estrés por sexo se determinó mediante la prueba de Mann-Whitney. La asociación entre las dimensiones de estrés y el rendimiento académico se verificó mediante la prueba $r$ de Pearson.

El programa SPSS v. 16.0 se utilizó para el procesamiento y análisis de datos. La significación estadística se estableció con $p<0,05$. 
Tabla III. Fuentes de estrés percibidas durante la formación de pregrado en odontología (cont.).

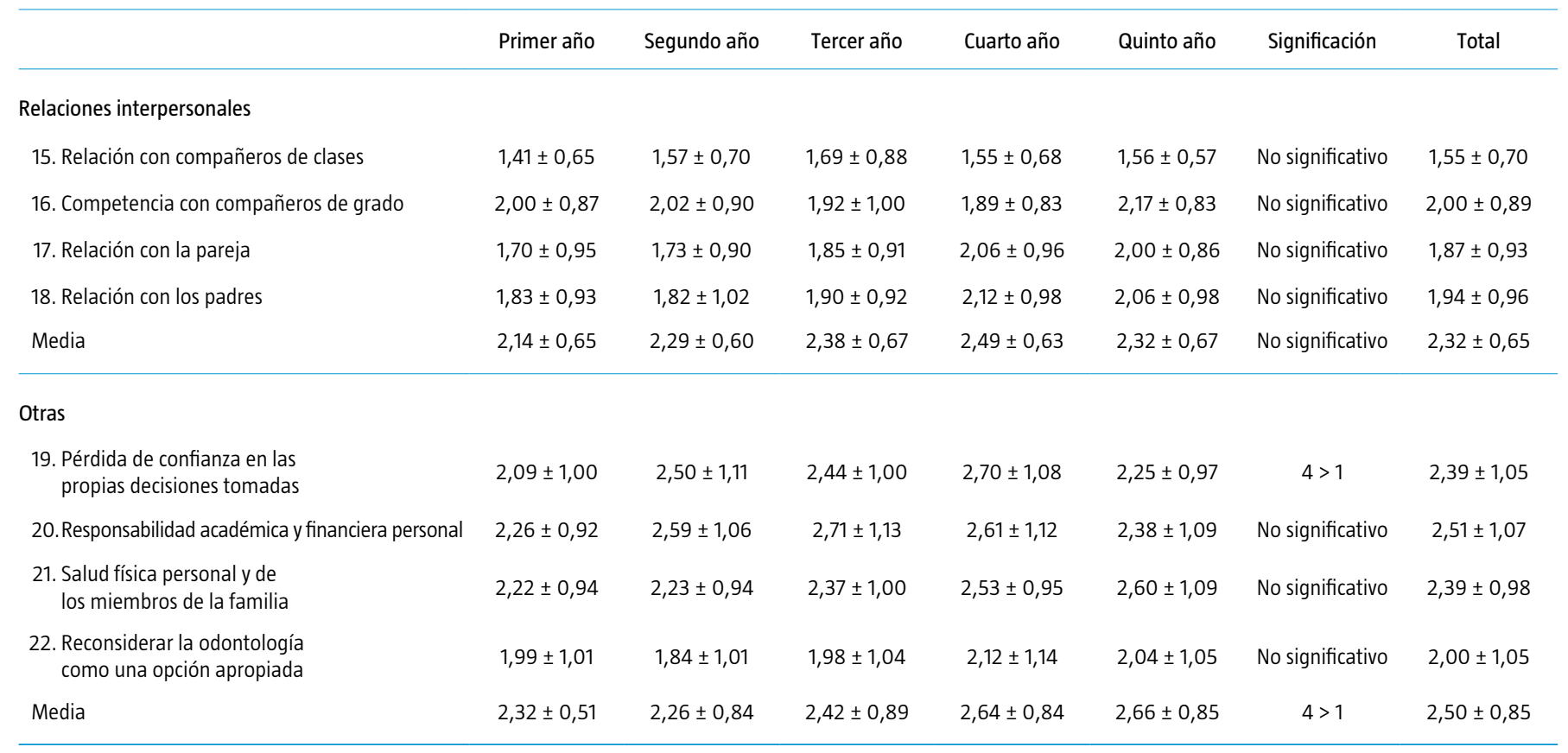

\section{Resultados}

De un total de 302 encuestados, $180(59,60 \%)$ eran mujeres y 122 (40,39\%) eran varones (Tabla II), con una edad media de 21,29 años. De primer a tercer año, la edad media no superaba los 21 años, pero en el quinto curso, la media alcanzó 25 años. El rango de edad de la muestra total fue de 18-33 años.

Como se muestra en la tabla III, las más importantes fuentes de estrés percibidas por los estudiantes de primer año fueron las siguientes: calificaciones y exámenes, miedo a fallar en un curso o un año, falta de tiempo para relajarse y dificultades en la comprensión de las clases. Un resultado similar se observó en los estudiantes de segundo año, excepto en el caso de la comprensión de lecturas, lo que muestra una menor puntuación. Para los estudiantes de tercer año, los elementos que se perciben como más estresantes fueron los mismos que para los años anteriores, con el agregado de las dificultades en la generación de habilidades manuales de precisión, el ambiente negativo creado por los supervisores clínicos y el elevado número de clases. Los estudiantes de cuarto año agregaron los temores sobre asumir responsabilidades en la atención de pacientes, el aprendizaje de procedimientos clínicos, los temores de que los laboratorios dentales se retrasen en la entrega de los trabajos solicitados y los temores asociados con la ausencia de los supervisores clínicos. Durante el quinto año, los estresores percibidos fueron los mismos que en el último año, aunque en diferente orden de importancia. También se consideró la escasa capacidad financiera de los pacientes para pagar sus tratamientos como un elemento estresor.

En todos los años, las fuentes que promediaron un estrés moderado a grave, excluyendo elementos clínicos, fueron las siguientes: las calificaciones y los exámenes, el miedo a fallar un curso y la falta de tiempo para relajarse. En el tercer año, cuando se inicia el trabajo clínico, la dificultad de generar habilidades manuales de precisión se consideró moderadamente estresante, así como el ambiente negativo creado por los supervisores clínicos en los años tercero, cuarto y quinto. Ninguna pregunta obtuvo una puntuación que se considerara extremadamente estresante.

Los resultados muestran (Tabla IV) que, entre el cuarto y el tercer año, las horas declaradas para ser usadas en estudio excede las establecidas en el plan de estudios oficial, en un rango del 10,4-48,2\%.

Las mujeres mostraron niveles más altos de estrés en todas las dimensiones; sin embargo, hubo una diferencia significativa solamente en 'carga de trabajo,' 'formación clínica' y 'otras' (Tabla V). 
Tabla IV. Diferencias entre el número de horas por semana dedicadas a actividades preclínicas y clínicas y las definidas en el plan de estudios original.

\begin{tabular}{lccccc}
\hline & Mínimo & Real & $\begin{array}{c}\text { Media } \pm \\
\text { desviación estándar }\end{array}$ & $\begin{array}{c}\text { Plan de } \\
\text { estudios }\end{array}$ & Diferencia \\
\hline Segundo año & 4 & 9 & $5,86 \pm 1,15$ & 4 & $+46,5 \%$ \\
\hline Tercer año & 10 & 22 & $16,36 \pm 3,31$ & 11 & $+48,2 \%$ \\
\hline Cuarto año & 11 & 38 & $24,29 \pm 8,90$ & 22 & $+10,4 \%$ \\
\hline Quinto año & 5 & 31 & $18,92 \pm 7,25$ & 15 & $+26,1 \%$ \\
\hline
\end{tabular}

Como se muestra en la tabla VI, el rendimiento académico presentó un amplio rango de valores $(3,7-6,1)$, con una media global de 5,03. Se observó la más amplia gama de calificaciones en los estudiantes de cuarto año. El rendimiento académico medio de los estudiantes de todos los cursos fue de alrededor de 5,0, sin diferencias significativas entre cursos.

Los resultados mostraron sólo dos correlaciones significativas entre las dimensiones de estrés y rendimiento académico de todos los cursos, uno positivo con la formación preclínica y clínica $(0,203)$ y uno negativo con la dimensión 'carga de trabajo' $(-0,148)$.

El rendimiento académico durante el quinto año de estudio tuvo una correlación negativa significativa con la dimensión de 'relaciones interpersonales' $(-0,381)$. Entre los estudiantes de primer año, en la dimensión 'otro' se encontró una correlación negativa $(-0,309)$ entre el rendimiento académico y aquellos elementos para 'considerar la odontología como una opción profesional adecuada' y 'falta de confianza en sí mismo', y entre los estudiantes de segundo año, una correlación negativa entre el rendimiento académico y la partida de 'responsabilidades financieras personales y académicas'. El rendimiento académico de los estudiantes de quinto año se correlacionó negativamente con los elementos 'responsabilidades financieras personales y académicas' y 'preocupaciones para los miembros personales,' 'preocupaciones personales' y 'otras relacionadas a la salud física familiar'.

\section{Discusión}

Las principales fuentes de estrés percibidas por los estudiantes de odontología fueron los exámenes y las calificaciones, el miedo a fallar en un curso o un año, la falta de tiempo para relajarse y la atmósfera creada por los supervisores clínicos. Estas fuentes principales de estrés son coherentes con las conclusiones de varios estudios realizados en diferentes países [1-6,10,13,14,18-20,22].

Durante el proceso de formación preclínica (segundo y tercer año), se agrega una serie de factores de estrés de los mencionados anteriormente, como las dificultades en la adquisición de habilidades manuales de precisión, las dificultades en el aprendizaje de los protocolos clínicos, la atmósfera creada por los supervisores, la ausencia de los supervisores, la respuesta del personal administrativo y asistentes dentales y el temor de que los laboratorios dentales puedan retrasar la entrega de trabajos. Todas estas fuentes de estrés pertenecen a las dimensiones de 'facultad y administración' y 'formación clínica.' En el cuarto y quinto año, el tratamiento de los pacientes y del personal docente y administrativo llegan a ser las principales fuentes de estrés.

El cuarto año presenta una situación única, con significativamente menor percepción de estrés en la dimensión 'carga de trabajo' que en los otros años, especialmente en el ítem 'elevado número de clases.' Este resultado se explica por el hecho de que el estudiante dedica casi todo su tiempo en el cuarto año a las actividades clínicas. Además, el factor de estrés 'falta de confianza en sí mismo' es más significativo en el primer año, y no aparece en los posteriores.

Los resultados asociados con la percepción de estrés en la relación de los estudiantes con el cuerpo docente y la administración y con respecto a los tratamientos de los pacientes revelan que los estudiantes en la etapa clínica de la formación tienen que hacer frente a dificultades no relacionadas con su compromiso de aprendizaje. Este estrés puede determinar futuras inseguridades en la realización de diversas actividades clínicas.

Una de las soluciones a este problema podría ser la indicada por Polychronopoulou y Divaris [18], quienes proponen iniciar el entrenamiento clínico muy cerca del comienzo de la carrera a fin de promover una respuesta de adaptación de estrés temprano entre los estudiantes. En este mismo contexto, Humphris et al [32] analizaron las fuentes percibidas de estrés académico en una muestra multinacional de estudiantes de odontología de Europa y concluyeron que el contacto temprano con los pacientes actuó como un factor protector en la manifestación de los síntomas de estrés.

En casi todos los años de estudio, las mediciones del tiempo medio utilizado por los alumnos en la realización de actividades académicas muestran una pronunciada tendencia a superar el tiempo estable- 
Tabla V. Comparación de niveles de estrés en cada dimensión (de acuerdo al rango medio) por sexo.

\begin{tabular}{lcccccc}
\hline & Carga de trabajo & $\begin{array}{c}\text { Entrenamiento } \\
\text { clínico }\end{array}$ & $\begin{array}{c}\text { Tratamiento } \\
\text { de pacientes }\end{array}$ & $\begin{array}{c}\text { Facultad y } \\
\text { administración }\end{array}$ & $\begin{array}{c}\text { Relaciones } \\
\text { interpersonales }\end{array}$ & Otras \\
\hline Hombres $(n=122)$ & 134,27 & 138,24 & 150,48 & 144,58 & 148,00 & 131,58 \\
\hline Mujeres $(n=180)$ & 163,18 & 160,49 & 152,19 & 156,19 & 153,87 & 165,00 \\
\hline Significación & 0,004 & 0,027 & 0,860 & 0,253 & 0,563 & 0,001 \\
\hline
\end{tabular}

cido por el plan de estudios, con la excepción de los estudiantes de cuarto y quinto año, que demuestran un promedio menor de horas de práctica que las establecidas en el plan de estudios oficial. Este promedio más bajo entre los estudiantes de cuarto y quinto año podría explicarse por las tasas de fracaso más altas en estos años que en los primeros años de formación; por tanto, los estudiantes que repiten cursos declaran menos horas dedicadas al trabajo clínico, lo que refleja una media más baja de número de horas que las efectivamente empleadas por los estudiantes de cuarto y quinto año.

En este estudio, las mujeres percibieron significativamente más estrés que los hombres en las preguntas que pertenecen a las dimensiones 'carga de trabajo,' 'formación clínica' y 'otros. Estos resultados son consistentes con otros estudios, lo que indica que el miedo a fallar en un curso o año y los exámenes son más estresantes en mujeres que en hombres $[6,14,18,33]$. Los niveles significativamente más elevados de estrés en las mujeres se pueden explicar por los diferentes patrones de respuesta a eventos estresantes por sexo, según Sanders y Lushington [21]. Estos autores también sugirieron que las diferencias en la intensidad de la respuesta a factores de estrés percibidos podrían explicarse por los distintos patrones de morbilidad psicológica y la construcción social de la masculinidad, en la que los hombres son menos expresivos acerca de sus preocupaciones.

Por el contrario, los estudios de Acharya [1] y de Kumar et al [22] observaron que los hombres mostraron mayores niveles de estrés que las mujeres. Estos estudios se llevaron a cabo entre los estudiantes de la India. Tales resultados podrían indicar una diferencia cultural en las respuestas de estrés relacionados con el género.

Otros autores no han hallado diferencias en el estrés percibido por sexo $[4,21,28]$. Gorter et al [34] afirmaron que el sexo no tiene papel alguno en la percepción de estrés y que las diferencias encontradas se pueden justificar por las influencias sociales.
Al observar la relación entre las diferentes dimensiones de estrés y el rendimiento académico, se puede establecer que este último se ve influido negativamente por la carga de trabajo académico y positivamente por el entrenamiento clínico.

Las correlaciones entre las fuentes de estrés y el rendimiento académico no son las mismas para cada año de estudio. En primer y cuarto año, la carga de trabajo tiene un efecto negativo, aunque débil, en la correlación con el rendimiento académico; sin embargo, a los cinco años, la dimensión 'relaciones interpersonales' se relaciona negativamente con el rendimiento académico. En los estudiantes de segundo y tercer año, no existe una correlación entre las variables.

La dimensión 'otro' debe analizarse por separado debido a que sus componentes son diferentes de los criterios utilizados para establecer las dimensiones anteriores. Entre los estudiantes de primer año, existe una correlación negativa entre la dimensión 'otro' y el rendimiento académico. El análisis de los diferentes ítems incluidos en la dimensión 'otro' entre los estudiantes de primer año muestra correlaciones negativas entre 'reconsiderar la odontología como una opción profesional adecuada' y 'falta de confianza en sí mismo' con el rendimiento académico. Estas correlaciones negativas podrían explicarse por el hecho de que los estudiantes que empiezan la universidad por primera vez podrían tener problemas para lidiar con los nuevos requisitos académicos, generando una crisis vocacional.

El plan de estudios no considera todas las actividades relacionadas con la práctica clínica, como las referidas al laboratorio dental, esterilización de instrumentos y completar la historia dental del paciente. Estas actividades exigen que los estudiantes dediquen más tiempo del establecido oficialmente para llevar a cabo todo el que se asocia con la atención de alta calidad del paciente. Por tanto, el plan de estudios debe reformularse en términos de horas establecidas para cada curso. 
Tabla VI. Distribución de la media de rendimiento académico por año de estudio.

\begin{tabular}{lcccc}
\hline & Mínimo & Máximo & Media & $\begin{array}{c}\text { Desviación } \\
\text { estándar }\end{array}$ \\
\hline Primer año & 3,80 & 5,70 & 4,86 & 0,41 \\
\hline Segundo año & 4,20 & 5,90 & 5,18 & 0,37 \\
\hline Tercer año & 3,90 & 5,70 & 5,11 & 0,39 \\
\hline Cuarto año & 3,70 & 6,10 & 4,91 & 0,48 \\
\hline Quinto año & 4,10 & 6,00 & 5,26 & 0,37 \\
\hline Total & 3,70 & 6,10 & 5,03 & 0,44 \\
\hline
\end{tabular}

Se revelaron una serie de cuestiones administrativas, como problemas con el personal auxiliar y falta de supervisores clínicos. Estos problemas deben estudiarse y resolverse en un futuro próximo por medio de un programa especial de formación para las personas involucradas.

Parece existir la necesidad de formar consejeros estudiantiles disponibles con el fin de evitar tensión, con la consiguiente alteración de la calidad de vida. Además, y lo más importante, debe ponerse en práctica un estudio completo destinado a la reformulación del plan de estudios, teniendo en cuenta la importancia de iniciar la atención al paciente en las primeras etapas de la carrera, ya que podría prevenir el estrés y sus efectos en los estudiantes.

Las escuelas de odontología deben continuar los estudios sobre aquellos factores que contribuyen al estrés entre sus estudiantes, considerando dichos factores en la construcción y evaluación de los planes de estudios.

\section{Bibliografía}

1. Acharya S. Factors affecting stress among Indian dental students. J Dent Educ 2003; 67: 1140-8.

2. Harikiran AG, Srinagesh J, Nagesh KS, Sajudeen N. Perceived sources of stress amongst final year dental under graduate students in a dental teaching institution at Bangalore, India: a cross sectional study. Indian J Dent Res 2012; 23: 331-6.

3. Divaris K, Lai CS, Polychronopoulou A, Eliades T, Katsaros C. Stress and burnout among Swiss dental residents. Schweiz Monatsschr Zahnmed 2012; 122: 610-5.

4. Sugiura G, Shinada K, Kawaguchi Y. Psychological well-being and perceptions of stress amongst Japanese dental students. Eur J Dent Educ 2005; 9: 17-25.

5. Pöhlmann K, Jonas I, Ruf S, Harper W. Stress, burnout and health in the clinical period of dental education. Eur J Dent Educ 2005; 9: 78-84.

6. Westerman GH, Grandy TG, Ocanto RA, Erskine CG. Perceived sources of stress in the dental school environment. J Dent Educ 1993; 57: 225-31.
7. Kalkwarf KL, Haden K, Valachovic RW. ADEA Commission on Change and Innovation in Dental Education. J Dent Educ 2005; 69: 1085-7.

8. Divaris K, Barlow P, Chendea S, Cheong W, Dounis A, Dragan I, et al. The academic environment: the students' perspective. Eur J Dent Educ 2008; 12: 120-30.

9. Davis E, Tudesco L, Meier S. Dental student stress, burnout, and memory. J Dent Educ 1989; 53: 193-5.

10. Sanders A, Lushington K. Effect of perceived stress on student performance in dental school. J Dent Educ 2002; 66: 75-81.

11. Lazarus R, Folkman S. Estrés y procesos cognitivos. 1 ed. Barcelona: Martínez Roca; 1986.

12. Alzahem AM, Van der Molen HT, Alaujan AH, Schmidt HG, Zamakhshary MH. Stress amongst dental students: a systematic review. Eur J Dent Educ 2011; 15: 8-18.

13. Silverstein ST, Kritz-Silverstein D. A longitudinal study of stress in first-year dental students. J Dent Educ 2010; 74: 836-48.

14. Garbee WH Jr, Zucker SB, Selby GR. Perceived sources of stress among dental students. J Am Dent Assoc 1980; 100: 853-7.

15. Lloyd D, Musser LA. Psychiatric symptoms in dental students. J Nerv Ment Dis 1989; 177: 61-9.

16. Goldstein MB. Sources of stress and interpersonal support among first-year dental student. J Dent Educ 1979; 43: 625-9.

17. Rajab LD. Perceived sources of stress among dental students at University of Jordan. J Dent Educ 2001; 65: 232-41.

18. Polychronopoulou A, Divaris K. Perceived sources of stress among Greek dental students. J Dent Educ 2005; 69: 687-92.

19. Muirhead V, Locker D. Canadian dental students' perceptions of stress. J Can Dent Assoc 2007; 73: 323.

20. Muirhead V, Locker D. Canadian dental students' perceptions of stress and social support. Eur J Dent 2008; 12: 144-8.

21. Sanders A, Lushington K. Sources of stress for Australian dental students. J Dent Educ 1999; 63: 688-97.

22. Kumar S, Dagli RJ, Mathur A, Jain M, Prabu D, Kulkarni S. Perceived sources of stress amongst Indian dental students. Eur J Dent Educ 1999; 13: 39-45.

23. Sofola O, Jeboda S. Perceived sources of stress in Nigerian dental students. Eur J Dent Educ 2006; 10: 20-3.

24. Morse Z, Dravo U. Stress levels of dental students at the Fiji School of Medicine. Eur J Dent Educ 2007; 11: 99-103.

25. Caldera J, Pulido B, Martínez M. Niveles de estrés y rendimiento académico en estudiantes de la carrera de psicología del centro universitario Los Altos. Revista de Educación y Desarrollo 2007; 7: 77-82.

26. Linn B, Zeppa R. Stress in junior medical students: relationship to personality and performance. J Med Educ 1984; 59: 7-12.

27. Bermúdez S, Durán M, Escobar C, Morales A. Evaluación de la relación entre rendimiento académico y estrés en estudiantes de medicina. Med UNAB 2006; 9: 198-205.

28. El-Gilany AH, Amr M, Awadalla N, El-Khawaga AH. Stress among medical and law students in Mansoura, Egypt. Middle East Journal of Family Medicine 2008; 6: 31-6.

29. Abdulghani HM Stress and depression among medical students: a cross sectional study at a medical college in Saudi Arabia. Pak J Med Sci 2008; 24: 12-7.

30. Stewart S, Lam T, Betson C, Wong C, Wong A. A prospective analysis of stress and academic performance in the first two years of medical school. Med Educ 1999; 33: 243-50.

31. Román C, Ortiz F, Hernández Y. El estrés académico en estudiantes latinoamericanos de la carrera de medicina. Revista Iberoamericana de Educación 2008; 46: 1-8.

32. Humphris G, Blinkhorn A, Freeman R, Gorter R, HoadReddick G, Murtomaa H, et al. Psychological stress in undergraduate dental students: baseline results from seven European dental schools. Eur J Dent Educ 2002; 6: 22-9.

33. Grandy T, Westerman G, Lupo J, Combs C. Stress symptoms among third-year dental students. J Dent Educ 1988; 52: 245-9.

34. Gorter R, Freeman R, Hammen S, Murtomaa H, Blinkhorn A, Humphris G. Psychological stress and health in undergraduate dental students: fifth-year outcomes compared with first-year baseline results from five European dental schools. Eur J Dent Educ 2008; 12: 61-8. 